\title{
Visualizations of Centre Vortex Structure in Lattice Simulations
}

\author{
James C. Biddle \\ Centre for the Subatomic Structure of Matter, Department of Physics, \\ The University of Adelaide, SA 5005, Australia \\ E-mail: james.biddledadelaide.edu. au

\section{Waseem Kamleh} \\ Centre for the Subatomic Structure of Matter, Department of Physics, \\ The University of Adelaide, SA 5005, Australia \\ E-mail: waseem.kamleh@adelaide.edu.au

\section{Derek B. Leinweber*i} \\ Centre for the Subatomic Structure of Matter, Department of Physics, \\ The University of Adelaide, SA 5005, Australia \\ E-mail: derek. leinweber@adelaide.edu.au
}

The structure of centre vortices in $S U(3)$ gauge-field configurations is examined through modern visualization techniques. Centre vortices are identified through gauge transformations maximizing the centre of the gauge group. Focusing on the thin vortices identified by Wilson loops having a non-trivial centre phase, the vortex structure is illustrated through renderings of oriented spatial plaquettes. Time oriented plaquettes are illustrated by identifying spatial links associated with these non-trivial plaquettes. The results illustrate an important role for the branching of $S U(3)$ centre vortices, possibly through monopole anti-monopole dynamics. Of particular interest is the correlation of the vortex structure and the topological-charge structure of the gauge fields, vital to dynamical chiral symmetry breaking and its associated mass generation. Singular points, where the projected vortex structure contains non-trivial centre phases in associated plaquettes spanning all four dimensions, are observed within the regions of nontrivial topological charge density calculated on the original Monte-Carlo generated configurations. The results provide new insights into the role of centre vortices in underpinning non-trivial topology in gauge fields. They reveal how the removal of centre-vortices necessarily destroys non-trivial topology and destabilizes would-be instantons under smoothing algorithms. The observed correlations further strengthen the idea that centre vortices are the seeds of dynamical chiral symmetry breaking.

The 36th Annual International Symposium on Lattice Field Theory - LATTICE2018

22-28 July, 2018

Michigan State University, East Lansing, Michigan, USA.

\footnotetext{
* Speaker.

$\dagger$ DBL thanks Michael Engelhardt for interesting and helpful conversations at Lattice 2018. The authors wish to thank Daniel Trewartha for his contributions to the gauge ensembles underlying this investigation. This research is supported with supercomputing resources provided by the Phoenix HPC service at the University of Adelaide and the National Computational Infrastructure (NCI) supported by the Australian Government. This research is supported by the Australian Research Council through Grants No. DP140103067, DP150103164, and LE160100051.
} 


\section{Introduction}

The essential, fundamentally-important, nonperturbative features of the QCD vacuum fields are: the dynamical generation of mass through chiral symmetry breaking, and the confinement of quarks. But what is the fundamental mechanism of QCD that underpins these phenomena? What aspect of the QCD vacuum causes quarks to be confined? Which aspect is responsible for dynamical mass generation? Do the underlying mechanisms share a common origin?

Recent research is now exposing the centre-vortex structure of nonperturbative gluon-field configurations to be the most fundamental aspect of nonperturbative vacuum structure giving rise to both confinement and dynamical chiral-symmetry breaking.

Removal of $S U$ (3) centre vortices removes confinement, while consideration of the vortices alone provides confinement [1]. Also, the planar vortex density of centre-vortex degrees of freedom scales with the lattice spacing providing an well defined continuum limit [1]. Similarly, removal of vortices suppresses the infrared enhancement of the gluon propagator. Again the vortices alone contain the long distance structure of the gluon fields responsible for the well-known infrared enhancement [2]. Studies of the nonperturbative quark propagator of the overlap-Dirac fermion operator further strengthen the fundamental role of centre vortices. A connection between center vortices and instantons was established through gauge-field smoothing [3] and evidence that centre vortices underpin dynamical chiral symmetry breaking in $S U(3)$ gauge theory was reported in Ref. [4]. Moreover the removal of centre vortex degrees of freedom from the gluon fields restores chiral symmetry [5]. Centre vortices are the seeds of dynamical chiral symmetry breaking.

In light of the importance of these most fundamental aspects of QCD vacuum structure, we will present visualizations of the complex structures formed by the projected centre vortices in $S U(3)$ gauge theory and explore their correlation with the topological charge density of the gluon fields.

\section{Centre Vortex Identification}

Centre vortices are identified through a gauge fixing procedure designed to bring the lattice link variables as close as possible to the identity multiplied by phase equal to one of the three cube-roots of one.

Here, vortices are identified by gauge fixing the original Monte-Carlo generated configurations directly to Maximal Centre Gauge [6, 7, 1], without any preconditioning [8]. The links $U_{\mu}(x)$ are gauge transformed to be brought close to the centre elements of $S U(3)$,

$$
Z=\exp \left(2 \pi i \frac{m}{3}\right) \mathbf{I}, \text { with } m=-1,0,1 \text {. }
$$

On the lattice this is implemented by searching for the gauge transformation $\Omega$ such that,

$$
\sum_{x, \mu}\left|\operatorname{tr} U_{\mu}^{\Omega}(x)\right|^{2} \stackrel{\Omega}{\rightarrow} \max .
$$

One then projects the gluon field to a centre-vortex only configuration where each link is a number (one of the roots of unity) times the identity matrix

$$
U_{\mu}(x) \rightarrow Z_{\mu}(x) \text { where } Z_{\mu}(x)=\exp \left(2 \pi i \frac{m_{\mu}(x)}{3}\right) \mathbf{I},
$$



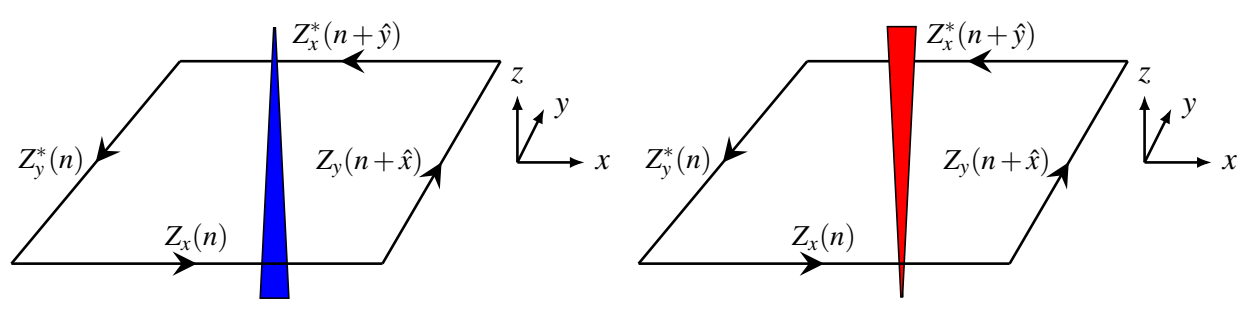

Figure 1: Rendering the centre charge of Eq. (2.4) associated with a plaquette in the $x-y$ plane at lattice site $n$. (left) An $m=+1$ vortex with centre charge $z=\exp (2 \pi i / 3)$ is rendered as a blue jet pointing in the $+\hat{z}$ direction. (right) An $m=-1$ vortex with centre charge $z=\exp (-2 \pi i / 3)$ is rendered as a red jet in the $-\hat{z}$ direction.

where $m_{\mu}(x)=-1,0,1$.

The vortices are identified by the centre charge, $z$, found by taking the product of the links around a plaquette,

$$
z=\prod_{\square} Z_{\mu}(x)=\exp \left(2 \pi i \frac{m}{3}\right) .
$$

A right-handed ordering of the dimensions is selected in calculating the centre charge. If $z=1$, no vortex pierces the plaquette. If $z \neq 1$ a vortex with charge $z$ pierces the plaquette. Vortices can be removed from the original gauge-field configuration by making the transformation

$$
U_{\mu}(x) \rightarrow U_{\mu}^{\prime}(x)=Z_{\mu}^{*}(x) U_{\mu}(x)
$$

\section{Centre Vortex Visualization Methods}

\subsection{Spatially Oriented Projected Centre Vortices}

As discussed in the previous section, vortex directions are indicated using a right-handed coordinate system. For example, with reference to Eq. (2.4), an $m=+1$ vortex in the $x-y$ plane is plotted in the $+\hat{z}$ direction as a blue jet. Similarly, an $m=-1$ vortex in the $x-y$ plane is plotted in the $-\hat{z}$ direction as a red jet. Figure 1 provides an illustration of this assignment.

Because the centre charge transforms to its complex conjugate under permutation of the two dimensions describing the plaquette, the centre charge can be thought of as a directed flow of charge $z=\exp (2 \pi i / 3)$. With our assignments above, the propagation of charge $z=\exp (-2 \pi i / 3)$ is in the direction opposite to the rendered jet. Noting that $\exp (-2 \pi i / 3)$ is equivalent to two units of charge $\exp (2 \pi i / 3)$, the propagation of two units of centre charge is also in a direction opposite to the rendered jet. This ambiguity makes it difficult to differentiate between vortex branching and monopole contributions [9].

\subsection{Space-Time Oriented Projected Centre Vortices}

In addition to purely spatial plaquettes, every link in the spatial volume has a forward and backward time-oriented plaquette associated with it. Thus, the three jets associated with the spatial $x-y, y-z$ and $z-x$ plaquettes, are complemented by jets in the three forward-time $x-t, y-t$ and $z-t$ plaquettes, and jets in the three backward-time $x-t, y-t$ and $z$ - $t$ plaquettes. 

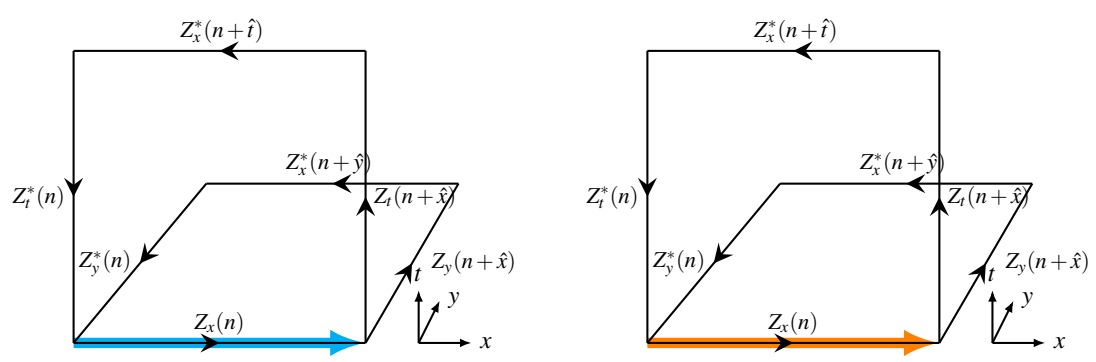

Figure 2: Rendering the centre charge of Eq. (2.4) associated with a space-time plaquette at lattice site $n$. An $m=+1$ vortex with centre charge $z=\exp (2 \pi i / 3)$ is rendered as a cyan link in the spatial volume (left) whereas an $m=-1$ vortex with centre charge $z=\exp (-2 \pi i / 3)$ is rendered as an orange link in the spatial volume (right). The directions of the arrow rendered indicate whether the space-time plaquette is forward or backward in time. Here the arrows point in the $+\hat{x}$ direction as a forward-looking plaquette is under consideration.

As the previous rendering technique cannot be applied to the hidden time dimension, spacetime oriented $\mathrm{P}$ vortices are illustrated by rendering the link in the spatial three-volume associated with the space-time $\mathrm{P}$ vortex. If a spatial link belongs to a $\mathrm{P}$ vortex in a space-time plaquette then the link is rendered in cyan for an $m=+1$ vortex and orange for an $m=-1$ vortex. In the spirit of the right-hand rule applied to the ordering of spatial-plaquette coordinates, the ordering of space-time coordinates is taken with reference to the four-dimensional Levi-Civita tensor. To distinguish whether the space-time plaquette is forward or backward in time, the link is rendered as a positively-directed arrow for forward space-time plaquettes and as a negatively-directed arrow for backward space-time plaquettes.

\section{Centre Vortex Structure}

Projected centre vortices (P-vortices) are surfaces in four dimensional space-time, analogous to the centre line of a vortex in fluid dynamics that maps out a surface as it moves through time. As the surface cuts through the three-dimensional volume of our visualization, a P-vortex line mapping the flow of centre charge is rendered.

The structure of projected centre vortices associated with spatial plaquettes is illustrated ${ }^{1}$ in Fig. 3. Here, a $20^{3} \times 40$ lattice is considered for $S U$ (3) Lüscher-Weisz [10] mean-field improved gauge fields with $a=0.125 \mathrm{fm}$. Inspection of the vortices reveals the flow of centre charge, intersection points and a prevalence of branching points resembling monopole or anti-monopole contributions, where three jets emerge from or converge to a point.

Upon activating the image of Fig. 3 views of these P-vortex features can be accessed from the Right-click "Views" menu including an "Intersection Point," a "Monopole/Branch Example," an "Anti-Monopole/Branch Example" and a "Cluster of Vortex Branches."

Figure 3 displays only one-third of the vortex information associated with these spatial links. Figure 4 introduces the P-vortex information associated with space-time plaquettes. This new

\footnotetext{
${ }^{1}$ To interact with Figs. 3 and 4, open this pdf document in Adobe Reader 9 or later. Linux users should install Adobe acroread version 9.4.1, the last edition to have full 3D support. From the "Edit" menu, select "Preferences..." and ensure "3D \& Multimedia" is enabled and "Enable double-sided rendering" is selected.
} 


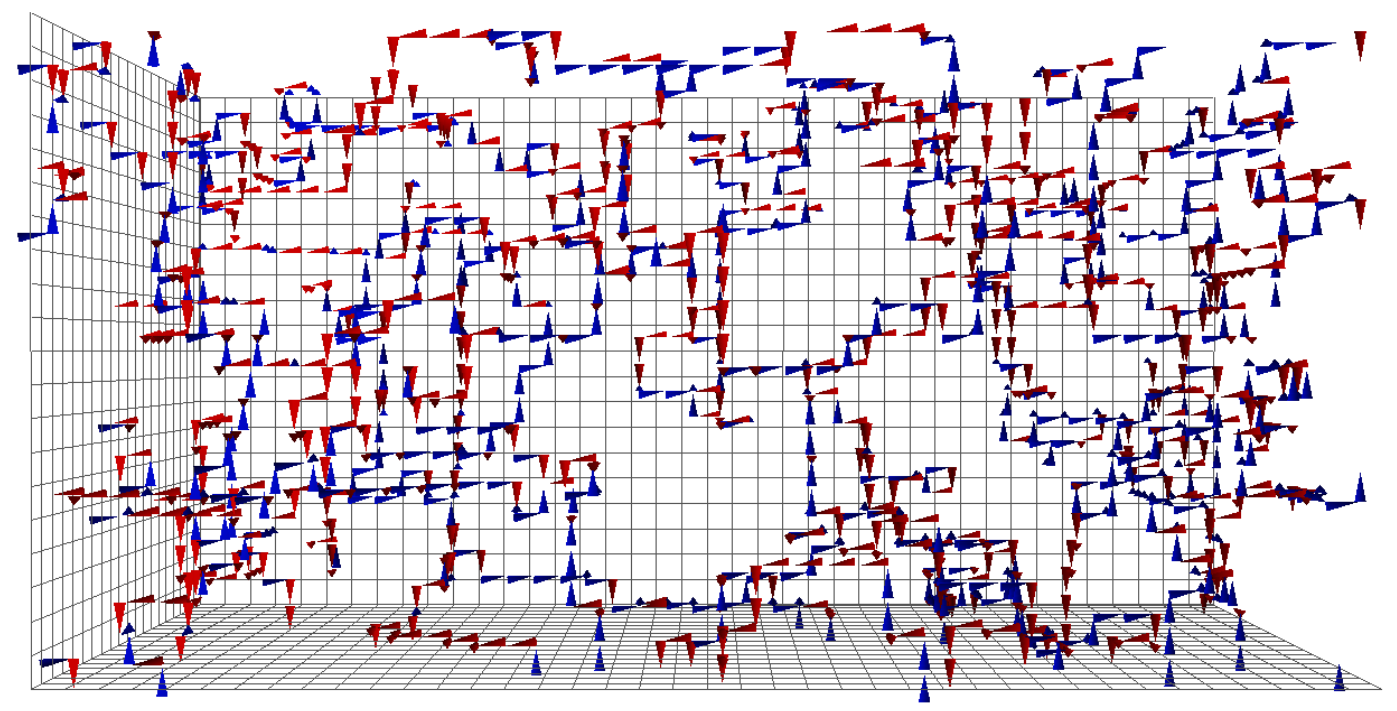

Figure 3: The projected centre-vortex structure of the gluon field. The flow of centre charge is illustrated by the jets as described in the text. (Click on the image to activate it in Adobe Reader. Click and drag to rotate, Ctrl-click to translate, Shift-click or mouse wheel to zoom. Right click to access the "Views" menu.)

information indicates the motion of vortex lines from one spatial slice in time to adjacent spatial slices in time, with forward (backward) pointing arrows indicating the direction of the vortex sheet as one moves forward (backward) in time.

It is possible to find sheets of links rendered as the vortex sheet runs approximately parallel to the time dimension. In this case the movement of the spatial jets can be over several lattice spacings as one moves from one time slice to the next. An example of this sheet of links is available in the right-click "Views" menu as "Vortex Sheet Between Spatial Planes."

Another point of interest is the presence of singular points [11] which can generate nontrivial topological charge density

$$
q(x)=\frac{g^{2}}{32 \pi^{2}} \varepsilon_{\mu \nu \rho \sigma} F_{\mu \nu}^{a b}(x) F_{\rho \sigma}^{b a}(x) .
$$

The presence of $\varepsilon_{\mu v \rho \sigma}$ indicates that the vortex surface must span all four dimensions at a particular lattice point. Singular points are points at which the tangent vectors of the vortex surface(s) span all four dimensions and can include touching points, intersection points and writhing points $[11,12]$. In the visualization of Fig. 4, singular points are lattice sites associated with both a rendered spatialplaquette jet and a rendered space-time plaquette link where the link is parallel to the jet. Figure 5 presents an example of a singular point. In short, one searches for a spatial plaquette with a jet, and a link on the corner parallel to the jet. The right-click "Views" menu of Fig. 4 contains examples 


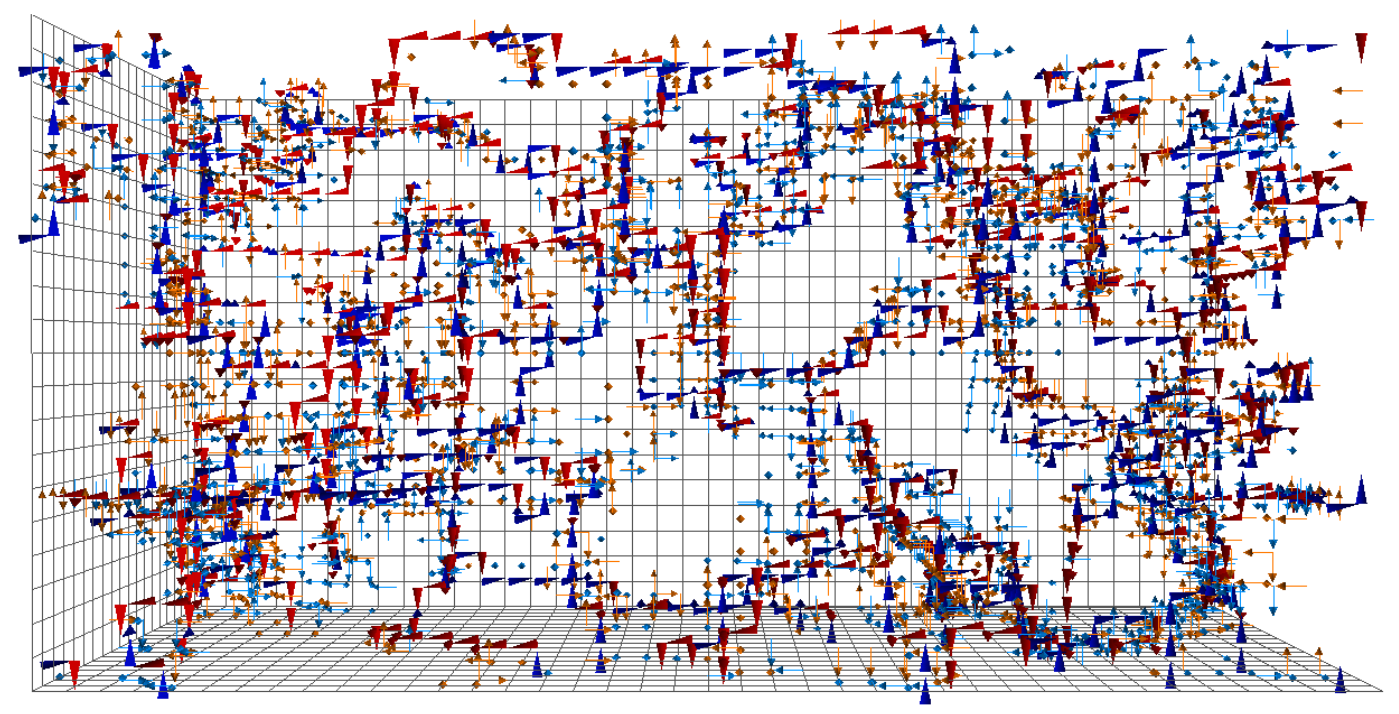

Figure 4: The centre-vortex structure of a gluon field configuration from both spatial and space-time oriented plaquettes. The flow of the centre charge of spatial plaquettes is illustrated by the jets and the spatial links of space-time oriented plaquettes, indicating the motion of the jets through time, are rendered in orange and cyan as described in the text. (Click to activate the image in Adobe Reader. Controls are summarised in the caption of Fig. 3.)

of a "Singular Point" and a "Collection of Singular Points."

Future work will present a correlation between these singular points and the topological charge density observed in the original configurations. It is common to see large complex vortex structures at the heart of regions having significant topoological charge density. Thus one now has a microscopic understanding of how the removal of centre-vortices via Eq. (2.5) necessarily destroys non-trivial topology and destabilizes would-be instantons under smoothing algorithms.

\section{Outlook}

The projected centre-vortex structure of $S U(3)$ gauge fields is complicated. The qualitative idea of a two-dimensional vortex sheet in four dimensions passing through a three-dimensional visualisation is realised. Perhaps the key new insight is the abundance of vortex branching/monopoleantimonopole dynamics.

Future directions of study include visualisations of Gribov-copy issues in vortex identification and explorations of the approach to the continuum limit. We are also interested in understand the impact of dynamical fermions [13] on centre-vortex structure. 


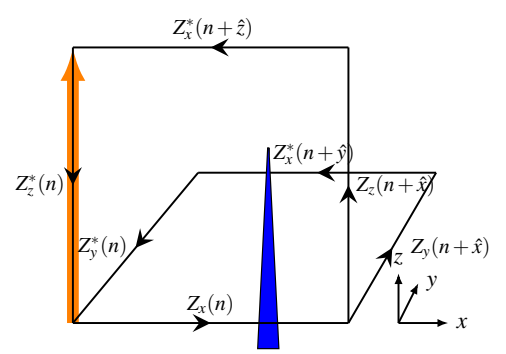

Figure 5: Signature of a singular point. Singular points are points at which the tangent vectors of the vortex surface(s) span all four dimensions. Thus, singular points are lattice sites associated with both a rendered spatial-plaquette jet and a rendered space-time plaquette link where the link is parallel to the jet. In this case, the blue jet is associated with centre charge in the $x-y$ dimensions and the orange link is associated with centre charge in the $z-t$ dimensions. Thus the vortex surface spans all four dimensions at site $n$.

\section{References}

[1] K. Langfeld, Vortex structures in pure SU(3) lattice gauge theory, Phys. Rev. D69 (2004) 014503, [hep-lat/0307030].

[2] J. C. Biddle, W. Kamleh, and D. B. Leinweber, Gluon propagator on a center-vortex background, Phys. Rev. D98 (2018), no. 9 094504, [arXiv: 1806.0430 ].

[3] D. Trewartha, W. Kamleh, and D. Leinweber, Connection between center vortices and instantons through gauge-field smoothing, Phys. Rev. D92 (2015), no. 7 074507, [arXiv: 1509.0551 ].

[4] D. Trewartha, W. Kamleh, and D. Leinweber, Evidence that centre vortices underpin dynamical chiral symmetry breaking in SU(3) gauge theory, Phys. Lett. B747 (2015) 373-377, [arXiv: 1502.0675 ].

[5] D. Trewartha, W. Kamleh, and D. Leinweber, Centre vortex removal restores chiral symmetry, J. Phys. G44 (2017), no. 12 125002, [arXiv:1708.0678].

[6] L. Del Debbio, M. Faber, J. Greensite, and S. Olejnik, Center dominance and Z(2) vortices in $S U(2)$ lattice gauge theory, Phys. Rev. D55 (1997) 2298-2306, [hep-lat/ 9610005 ].

[7] K. Langfeld, H. Reinhardt, and O. Tennert, Confinement and scaling of the vortex vacuum of SU(2) lattice gauge theory, Phys. Lett. B419 (1998) 317-321, [hep-lat/ 9710068 ].

[8] A. O’Cais, W. Kamleh, K. Langfeld, B. Lasscock, D. Leinweber, et al., Preconditioning Maximal Center Gauge with Stout Link Smearing in SU(3), Phys.Rev. D82 (2010) 114512, [arXiv:0807.0264].

[9] F. Spengler, M. Quandt, and H. Reinhardt, Branching of Center Vortices in SU(3) Lattice Gauge Theory, Phys. Rev. D98 (2018), no. 9 094508, [arXiv: 1810.0407 ].

[10] M. Luscher and P. Weisz, On-shell improved lattice gauge theories, Commun. Math. Phys. 97 (1985) 59, [Erratum-ibid. 98, 433 (1985)].

[11] M. Engelhardt, Center vortex model for the infrared sector of Yang-Mills theory: Topological susceptibility, Nucl. Phys. $\mathbf{B 5 8 5}$ (2000) 614, [hep-lat/ 0004013 ].

[12] M. Engelhardt, Center vortex model for the infrared sector of SU(3) Yang-Mills theory: Topological susceptibility, Phys. Rev. D83 (2011) 025015, [arXiv: 1008 .4953].

[13] W. Kamleh, P. O. Bowman, D. B. Leinweber, A. G. Williams, and J. Zhang, Unquenching effects in the quark and gluon propagator, Phys. Rev. D76 (2007) 094501, [arXiv: 0705. 4129]. 